$\Rightarrow$ GENE THERAPY

Hematopoietic stem cell gene therapy with a lentiviral vector in X-linked adrenoleukodystrophy

Cartier, N. et al. Science 326, 818-823 (2009)

$\mathrm{X}$-linked adrenoleukodystrophy (ALD) is a brain-demyelinating disease caused by a deficiency in ATP-binding cassette transporter D1 (ABCD1). In this gene therapy trial, autologous $\mathrm{CD} 34^{+}$cells were removed from two patients with ALD, genetically corrected ex vivo with a lentiviral vector encoding wild-type $A B C D 1$, and then re-infused into the patients after they had received myeloablative treatment. Haematopoietic stem cells were transduced in the patients and, 14-16 months after cell re-infusion, progressive cerebral demyelination had stopped.

\section{$\Rightarrow$ LEARNING AND MEMORY}

Restoration of norepinephrine-modulated contextual memory in a mouse model of Down syndrome

Salehi, A. et al. Science Transl. Med. 1, 7 ra17 (2009)

The mechanism by which the defects in contextual learning and memory that are associated with Down's syndrome affect the locus coeruleus (which innervates the hippocampus) is not well understood. Using a mouse model of Down's syndrome, Salehi and colleagues showed that, despite dysfunction and degeneration of locus coeruleus neurons, the post-synaptic target of the neurons - the $\beta 1$-adrenergic receptor — was upregulated and remained responsive to noradrenergic agonists. A prodrug of noradrenaline reversed defects in contextual learning, suggesting that such compounds could be beneficial in Down's syndrome.

\section{$\Rightarrow$ NEUROLOGICAL DISORDERS}

Role of NMDA receptor-dependent activation of SREBP 1 in excitotoxic and ischemic neuronal injuries

Taghibiglou, C. et al. Nature Med. 15, 1399-1406 (2009)

Balance between synaptic versus extrasynaptic NMDA receptor activity influences inclusions and neurotoxicity of mutant huntingtin

Okamoto, S. et al. Nature Med. 15, 1407-1413 (2009)

These two papers highlight how controlled regulation of the $\mathrm{N}$-methyl-D-aspartate receptor (NMDAR) might have beneficial effects in neurological disorders. Taghibiglou and colleagues showed that blocking NMDAR-dependent transcription can reduce the excitotoxic neuronal death that is associated with stroke. NMDAR-dependent activation of the transcription factor sterol regulatory element binding protein 1 (SREBP1) mediated neuronal cell death in response to ischaemia. The activation of SREBP1 resulted from increased degradation of insulin-induced gene 1 , the inhibition of which with an interference peptide reduced neuronal damage and improved behavioural outcome in a rat model of stroke. In the second study, Okamoto and colleagues showed that NMDAR activity can modulate aggregation of mutant huntingtin protein (mtHTT) in Huntington's disease. In cellular models, synaptic NMDAR activity was required for the formation of $\mathrm{mtHTT}$ inclusions. Treatment of transgenic mice with a low dose of the NMDAR channel blocker memantine blocked extrasynaptic NMDARs and ameliorated neuropathological and behavioural manifestations, whereas high-dose memantine - which blocks both extrasynaptic and synaptic NMDAR activity - decreased neuronal inclusions and worsened these outcomes.

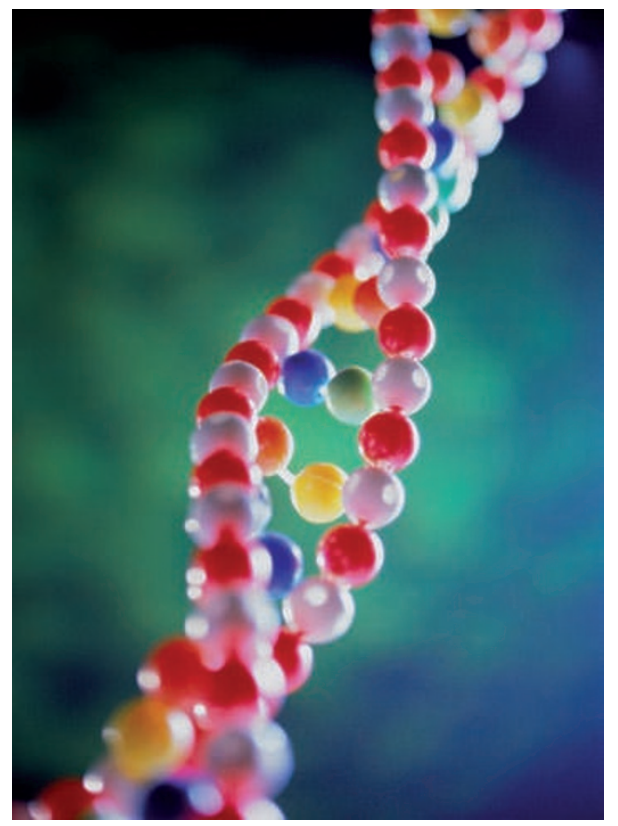

